SHORT REPORT

\title{
A novel mutation (V89L) in the presenilin 1 gene in a family with early onset Alzheimer's disease and marked behavioural disturbances
}

\author{
R Queralt, M Ezquerra, A Lleó, M Castellví; J Gelpí, I Ferrer, N Acarín, L Pasarín, \\ R Blesa, R Oliva
}

J Neurol Neurosurg Psychiatry 2002;72:266-269

A novel mutation (V89L) in the presenilin 1 (PSEN1) gene is described in a family with pathologically confirmed Alzheimer's disease. The mutation was identified in two affected members with early onset Alzheimer's disease characterised by early and marked behavioural disturbances. The mutation is located on the same side of the helix as other described mutations in the first transmembrane domain and its relation to other mutations in this helix suggests that they share a common pathogenic mechanism.

E arly onset familial Alzheimer's disease is genetically heterogeneous and three genes have been involved in this form of the disease, the amyloid precursor protein (APP) gene on chromosome $21,{ }^{1}$ the presenilin 1 (PSEN1) gene on chromosome $14,{ }^{2}$ and the presenilin 2 (PSEN2) gene on chromosome $1 .{ }^{34}$ Mutations in the PSEN 1 gene account for almost one half of the cases of early onset familial Alzheimer's disease. ${ }^{5}$ In this report we describe a novel PSEN1 mutation (V89L) associated with pathologically confirmed early onset familial Alzheimer's disease, with a phenotype characterised by early and marked behavioural disturbances. In addition, molecular modelling showed that the mutation was located within an array of mutations in transmembrane 1 of the presenilin gene and suggests that they may share a common pathogenic mechanism.

\section{PATIENTS AND METHODS \\ Subjects}

The family members studied belong to a two generation family with early onset familial Alzheimer's disease from Barcelona, Spain. The proband was member II-l (fig l A, table 1), a 47 year old pharmacist with a 1 year history of memory problems. His family also reported a 3 year history of frequent arguments at his job, paranoid thinking about his colleagues, and problems making easy decisions. He became more reserved and developed complex rituals such as writing inappropriately long notes, or shaking his clothes many times before dressing himself. Neurological examination showed a hemifacial spasm and his cognitive profile (explored using the Wechlser adult intelligence scale, mini mental state examination, and the Cambridge mental disorders of the elderly examination (CAMCOG) scales) showed severe impairment in recent verbal memory, especially in delayed recall while the remote memory was preserved. He also showed spatial and temporal disorientation, deficits in attention, and abstract thinking. A diagnosis of Alzheimer's disease was made. ${ }^{6}$ Two years later he developed apraxia, acalculia, word finding difficulty, abnormal prosody, and phonological errors. He died 5 years later, at the age of 52. A neuropathological examination confirmed the clinical diagnosis of Alzheimer's disease. Gross findings showed diffuse moderate cortical atrophy with an enlarged ventricular system. Microscopic examination showed neurofibrillary tangles and neuritic plaques with dystrophic neurites corresponding to a stage VI of Braak and Braak (fig l B). Neuritic plaques were most frequent in the hippocampus, amygdala, and neocortex. Neurofibrillary tangles were most abundant in the neocortex and hippocampus and were less abundant in the striatum, thalamus, and midbrain. Amyloid angiopathy was present in cortical blood vessels. Patient II-2 was a 50 year old mathematics teacher with a 1 year history of memory loss and problems in performing his class duties. His wife reported episodes of exhibitionism and masochistic conduct that resulted in him going to court. During the past 3 years he also had been anxious, dysphoric, irritable, and neglectful in his job and daily activities by contrast with his previous behaviour. The neurological examination was normal and his initial neuropsychological profile (explored with the Wechlser adult intelligence scale and mini mental state examination, Benton visual retention test, Rey-Osterrieth complex figure, and Wechsler memory scale) showed generalised cognitive impairment, especially in visuospatial tasks, attention, and verbal and visual memory function. Remote memory, naming, and comprehension were preserved at this moment. A new examination 3 years later showed word finding difficulty and reduced comprehension, poor abstract thinking, and visual agnosia. Brain MRI was normal. A diagnosis of Alzheimer's disease was made. ${ }^{6}$ At present he is alive and is dependent for most activities of daily living. Member II-3 was a 43 year old woman who had a normal neurological and neuropsychological examination. Member I-1 was a 58 year old ex-boat captain who was examined in 1970 because of memory loss. He had displayed a 7 year history of anxiety, apathy, and obsessive and paranoid thinking concerning a boat accident that occurred during his previous job. Three years later he began consuming large amounts of alcohol. The neuropsychological examination (conducted with ReyOsterrieth complex figure, Bender-Gestalt test, Wechsler memory scale) showed deficits in visuospatial tasks, memory, and conceptual tasks. He had deficits in delayed recall but improved substantially with recognition. Attention and concentration were clearly reduced and language was preserved. Two years later he became irritable, with psychomotor agitation, disorientation, and progressive aphasia and died in 1978, at the age of 66. After informed consent, blood samples were obtained from members II-1, II-2, and II-3 for genetic analysis.

Abbreviations: PSEN1, presenilin 1; PSEN2, presenilin 2; APP, amyloid precursor protein 
Table 1 Summary of the clinical picture of the family with the V89L mutation

\begin{tabular}{llll}
\hline Feature & I-1 & II-1 & II-2 \\
\hline Age of onset (y) & 51 & 46 & 49 \\
Age of death (y) & 66 & 52 & - \\
Duration (y) & 15 & 6 & - \\
Behavioural abnormalities & Anxiety, apathy, paranoid thinking & $\begin{array}{l}\text { Paranoid, obsessive behaviour, social } \\
\text { shyness } \\
\text { Neuropsychological domains affected }\end{array}$ \\
& Memory, visuospatial tasks, & Memory, attention, executive function & Visuospatial tasks attention, memory \\
Neuropathology & executive function & Stage VI Braak and Braak & - \\
PSENI mutation & NA & + & + \\
APOE genotype & NA & $\epsilon 3 \in 3$ & $\epsilon 2 \epsilon 3$ \\
\hline
\end{tabular}

*Data from the initial evaluation.

NA, not available.

\section{Genetic analysis}

Mutational analysis of the APP gene (exons 16 and 17) and the coding exons of the PSEN1 gene were performed through single strand comphormation polymorphism (SSCP) as described. ${ }^{17}$ Samples with abnormal mobility were subsequently sequenced using the ABI PRISM dye terminator cycle sequencing ready reaction kit (Perkin Elmer, Foster City CA, USA) with an automatic sequencer (ABI PRISM model 377).

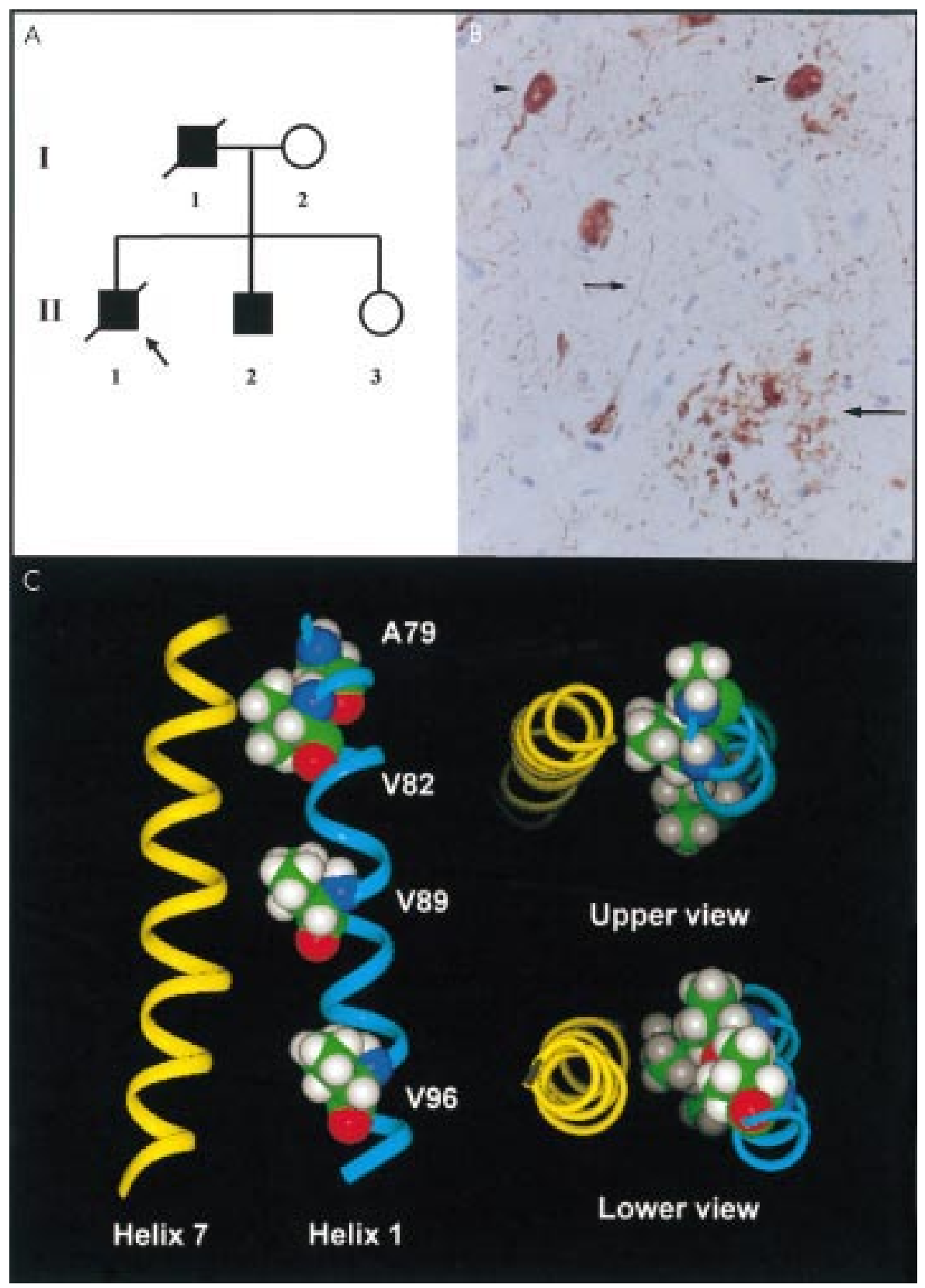

Figure 1 (A) Pedigree of the family with the V89L mutation associated with early onset Alzheimer's disease. Filled symbols correspond to patients with Alzheimer's disease and open symbols correspond to the unaffected members. The arrow indicates the proband. Members from generation III were excluded to protect their identities. (B) Neurons with neurofibrillary degeneration (arrowheads), neuropile threads (short arrows), and dystrophic neurites in a neuritic plaque (long arrow) in the subiculum. Paraffin section, tau immunohistochemistry, light haematoxylin counterstaining $\times 400$. (C) PSEN 1 modelling of helixes 1 and 7 indicating the positions of some of the described mutations in exon 4 (A79, V82, V89, V96). The model is based on the bacteriorhodopsin framework where two helices, 1 and 7 , have been mutated to the presenilin sequence. Views from the top and the bottom of the model are shown on the right. 
Restriction fragment length analysis of exon 4 was performed by MaeIII digestion. APOE was genotyped as described. ${ }^{8} \mathrm{~A}$ molecular modelling analysis was performed on a structural model based on the three dimensional structure of bacteriorhodopsin (reference 2BRD, protein data bank), ${ }^{9}$ with part of the sequence (residues 9-31 and 203-226) mutated to PSEN1 (corresponding residues 78 to 100 and 413 to 435, respectively). Model building and analysis were performed in a Silicon Graphics Power Indigo 2 workstation using the molecular modelling package Insight II, Homology and Discover (Biosym -MSI, San Diego, CA, USA) as previously described. ${ }^{10}$

\section{RESULTS}

Mutation analysis disclosed a heterozygous V89L mutation due to a G-T transition at the first position of codon 89 (GTG $\rightarrow$ TTG) in exon 4 of the PSEN l gene, which predicted a valine to leucine change. This mutation is located in the transmembrane 1, removes the MaeIII restriction site, and was not detected in 100 unrelated subjects ( 50 cognitively normal and 50 patients with Alzheimer's disease). Restriction analysis showed the presence of the mutation in the proband and member II-2 and its absence in the unaffected sister. The APOE genotypes were $\epsilon 33$ and $\epsilon 32$ in members II- 1 and II-2 respectively. The mutated residue is conserved in human PSEN1 and PSEN2 and in Spe4 from C elegans. ${ }^{3}$

To assess the possible effects of the V89L mutation and some of the described mutations in the transmembrane 1 (A79V, V82L, and V96F $)^{7112}$ we introduced these residues to a structural model of PSI based on the three dimensional structure of bacteriorhodopsin. Figure $1 \mathrm{C}$ shows a representation of helices 7 and 1 of the model. Assuming $\alpha$-helical structure for this transmembrane 1 , these mutations lie in the interface between the two $\alpha$-helices in a region where hydrophobic contact exists. The four mutated residues analysed (A79, V82, V89, V96) fall on the same side of the helix as would be expected as their separation in sequence (four or seven residues) coincides with helix periodicity. Orientation of helix 1 deduced from hydrophobic moments directs these four residues towards helix 7, especially residues 82,89 , and 96. A79 deviates slightly towards the centre of the protein. All valine residues are in close contact with hydrophobic residues of helix 7.

\section{DISCUSSION}

We report a novel missense mutation (V89L) in exon 4 of the PSENl gene. The cosegregation of the mutation with early onset familial Alzheimer's disease in this family, its absence in 200 analysed chromosomes, and the evolutionary conservation of this residue suggest that it is a pathogenic mutation. Until now five mutations located in the TMI of the PSEN1 have been reported in early onset familial Alzheimer's disease. ${ }^{71-14}$ The different distribution of these mutations and the similar age of onset in these families suggest that the major part of this PSENI coding region is functionally important.

The clinical picture of the affected members from this family was characterised by marked behavioural abnormalities. Although behavioural changes are ubiquitous in Alzheimer's disease, they usually show a more irregular temporal pattern than the cognitive decline. In our patients the personality disorder appeared early in the course of the disease, even before cognitive complaints. These behavioural changes added to the cognitive deficits may reflect an involvement of frontal or frontal-subcortical circuits. Indeed the presence of neurofibrillary tangles in the thalamus and striatum in member II-1 could support this idea. In addition, the contrast between the early behavioural changes and the subsequent executive dysfunction may indicate a differential involvement of these pathways.
To get some insight into the possible structural effects of the V89L mutation and other mutations located in the same exon of the PSENI gene, we built a partial model of its three dimensional structure. This approach has been previously performed. ${ }^{10}{ }^{15} 16$ Although the interpretation of the model is hypothetical, the mutation forms part of an array of mutations, all of them in the same face of the helix, and produces an increase in the size of the side chain. This would increase the distance between the two helices and would introduce an extra stress in the packing between helix 7 and 1 . In addition, all mutated residues are hydrophobic favouring a structural rather a functional role for these residues. As recently suggested, ${ }^{16}$ this supports the idea that this helical face is a region critical for the biological function which these mutations interfere with.

In conclusion, we have described a novel PSENI mutation characterised by early and marked behavioural abnormalities. In addition, the mutation indicates the presence of an array of mutations in the transmembrane 1 domain of the PS 1 pointing to a common pathogenic mechanism.

\section{ACKNOWLEDGEMENTS}

This work was supported in part by a grant from the Generalitat de Catalunya (SGR 1997-2000) to RO

\section{Authors' affiliations \\ R Queralt, M Ezquerra, R Oliva, Genetics Service, Hospital Clínic, Universitat de Barcelona, Spain \\ A Lleó, M Castellví, R Blesa, Neurology Service \\ I Ferrer, Bank of Neurological Tissues \\ N Acarín, Neurology Service, Hospital Vall d'Hebron. Barcelona, Spain \\ L Pasarín, Institut Psiquiatric, IMAS, Barcelona, Spain}

Correspondence to: Dr R Oliva, Genetics Service, Hospital Clínic, Villarroel 170, 08036 Barcelona, Spain; roliva@clinic.ub.es

Received 11 July 2001

In revised form 11 September 2001

Accepted 17 September 2001

\section{REFERENCES}

1 Goate A, Chartier-Harlin MC, Mullan M, et al. Segregation of a missense mutation in the amyloid precursor protein gene with familial Alzheimer's disease. Nature 1991;349:704-9.

2 Sherrington R, Rogaev El, Liang Y, et al. Cloning of a gene bearing missense mutations in early-onset familial Alzheimer's disease. Nature 1995;375:754-60

3 Levy-Lahad E, Wasco W, Poorkak P, et al. Candidate gene for the chromosome 1 familial Alzheimmer's disease locus. Science 1995:269:973-7.

4 Rogaev EI, Sherrington R, Rogaeva EA, et al. Familial Alzheimer's disease in kindreds with missence mutations in a gene on chromosome 1 related to the Alzheimer's disease type 3 gene. Nature 1995; 376:775-8

5 Rosenberg RN. The molecular and genetic basis of AD: the end of the beginning: the 2000 Wartenberg lecture. Neurology 2000;54: 2045-54.

6 McKhann G, Drachman D, Folstein MF, et al. Clinical diagnosis of Alzheimer's disease: report of the NINCDS-ADRDA-work group under the auspices of Department of Health and Human Services, Task Force. auspices of Department of Health

7 Cruts $M$, van Duijn CM, Backhovens $\mathrm{H}$, et al. Estimation of the genetic contribution of presenilin-1 and -2 mutations in a population-based study of presenile Alzheimer's disease. Hum Mol Genet 1998;7:43-51.

8 Blesa R, Adroer R, Santacruz P, et al. High apolipoprotein E epsilon 4 allele frequency in age-related memory decline. Ann Neurol 1996;39:515-8.

9 Bersntein FC, Koetzle TF, Williams GJ, et al. The proteins data bank: a computer-based archival file for macromolecular structures. J Mol Biol 1977;112:535-42

10 Ezquerra $M$, Carnero $C$, Blesa $R$, et al. A presenilin 1 mutation (Ser 169Pro) associated with early-onset AD and myoclonic seizures. Neurology 1999;52:566-70.

11 Finckh U, Muller-Thomsen T, Mann U, et al. High prevalence of pathogenic mutations in patients with early-onset dementia detected by sequence analyses of four different genes. Am J Hum Gene 2000;66: 110-7.

12 Kamino K, Sato S, Sakaki Y, et al. Three different mutations of presenilin 1 gene in early-onset Alzheimer's disease families. Neurosci Lett 1996;208: 195-8. 
13 Steiner $\mathbf{H}$, Revesz T, Neumann M et al. A pathogenic presenilin-1 deletion causes abberrant A-beta-42 production in the absence of congophilic amyloid plaques. J Biol Chem 2000;276:7233-9.

14 Jacquier $M$, Arango $D$, Torres $O$, et al. Presenilin mutations in a Colombian familial and sporadic AD sample. Neurobiol Aging 2000;21(supp 1):S176.
15 Crook R, Ellis R, Shanks M, et al. Early-onset Alzheimer's disease with a presenilin-1 mutation at the site corresponding to the Volga German presenilin-2 mutation. Ann Neurol 1997;42:124-8.

16 Hardy J, Crook R. Presenilin mutations line up along transmembrane $\alpha$-helices. Neurosci Lett 2001;306:203-5.

\section{NEUROLOGICAL PICTURE}

\section{Simultanagnosia in posterior cortical atrophy}

\begin{abstract}
$A^{54}$
54 year old woman presented with visual disturbance followed by geographical disorientation for 3 years. Neurological and neuropsychological examinations were remarkable for severe simultanagnosia accompanied by optic ataxia, oculomotor apraxia, and prosopagnosia without colour agnosia. Also, she had contructional and dressing apraxia, impaired naming, ideomotor apraxia, acalculia, and right-left disorientation with intact conversational speech and semantic word fluency. Although verbal and visual memories were compromised on formal memory tests, episodic memory in daily living was preserved. Brain ${ }^{18} \mathrm{~F}$-fluoro-2-deoxy-Dglucose PET showed marked hypometabolism involving bilateral temporoparietooccipital areas (figl A), which, together with clinical features, was consistent with posterior cortical atrophy.

Immediately after a calibration, each of two modified Navon figures (fig 1 B) was presented on the computer screen and the patient and a 53 year old female control were asked to find "star" on the number " 4 " composed of symbols (left of fig l B) and to find number " 4 " on a Korean letter (meaning "ball") composed of numbers (right of fig 1 B). With head and chin fixation, the subjects' left eye was monitored for 10 seconds after presentation of stimuli, using video-oculography (SMI, Germany), which allowed 60 measurements a second with resolution of $0.1^{\circ}$. The control scanned the whole stimuli (figl C), whereas the patient scanned only focal areas of the global figures (fig 1 D). Simultanagnosia may result from either deficient scanning ability or failure to organise pieces of visual information at the mental representational level despite intact scanning ability. Our patient's results may support the deficient scanning ability theory.
\end{abstract}

S J Yoon

Department of Neurology, Masan Samsung Hospital, Sungkyunkwan University School of Medicine, Masan, Korea

J M Park, D L Na

Department of Neurology, Samsung Medical Center, Sungkyunkwan University School of Medicine, Seoul, Korea

Correspondence to: $\operatorname{Dr}$ D L Na, Department of Neurology, Samsung Medical Center Sungkyunkwan University School of Medicine, 50 ILwon-dong Kangnam-ku, Seoul, 135-710 Korea; dukna@smc.samsung.co.kr
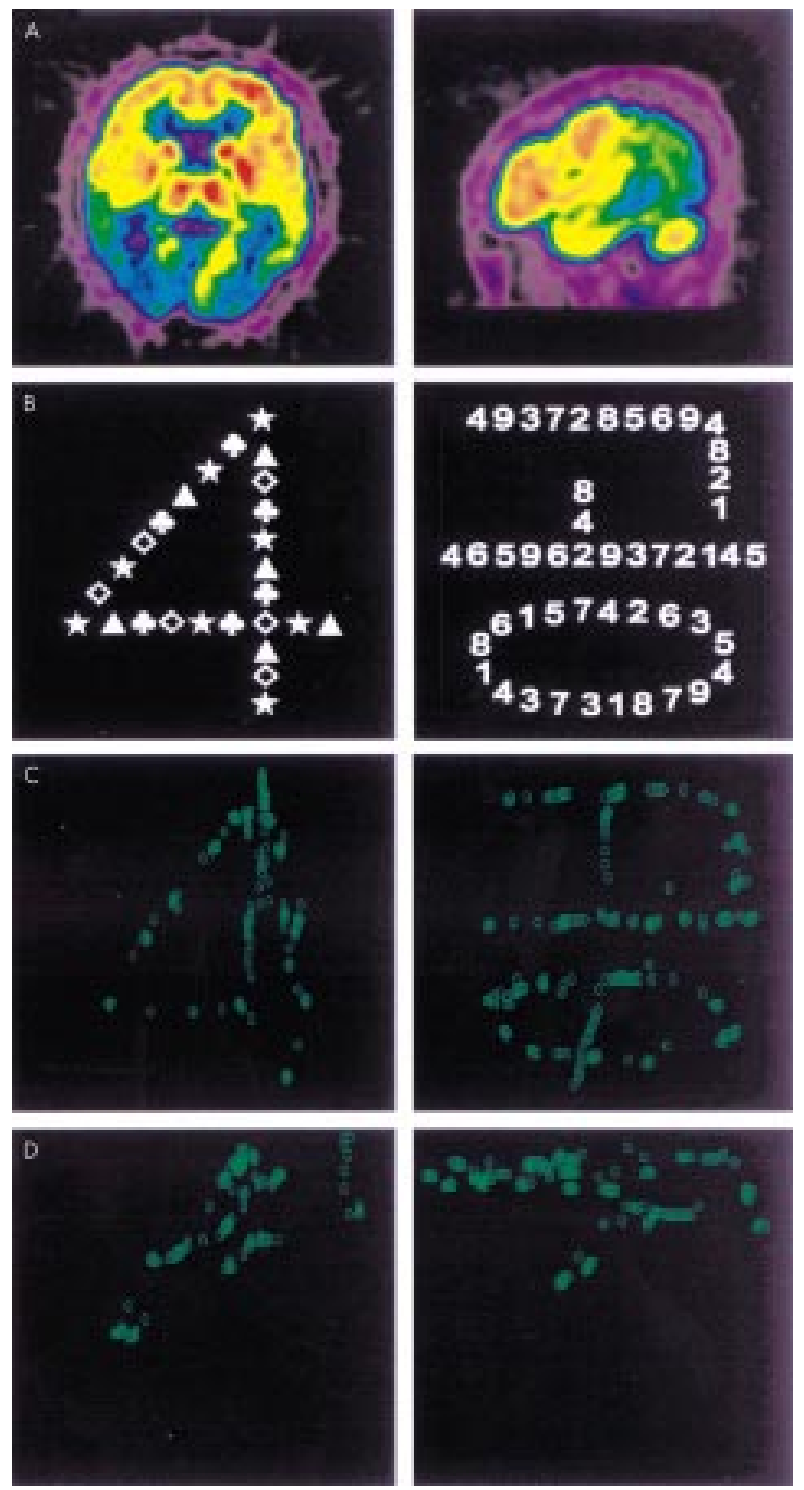

PET of patient and video-oculography for patient and a control. 